\title{
Design and Evaluation of a Wearable Assistive Technology for Hemianopic Stroke Patients
}

\author{
Navid Amini \\ Dept. of Computer Science \\ Cal. State Univ., Los Angeles \\ Los Angeles, USA \\ namini@calstatela.edu
}

\author{
Benjamin Braun \\ School of Medicine \\ Quinnipiac University \\ North Haven, USA \\ benjamin.braun@qu.edu
}

\author{
Jung S. Lim \\ Dept. of Computer Science \\ Cal. State Univ., Los Angeles \\ Los Angeles, USA \\ jlim34@calstatela.edu
}

\author{
Hassan Ghasemzadeh \\ School of EECS \\ Washington State University \\ Pullman, USA \\ hassan.ghasemzadeh@wsu.edu
}

\author{
Farnaz Mohammadi \\ Dept. of Bioengineering \\ UCLA \\ Los Angeles, USA \\ farnazmdi@ucla.edu
}

\author{
Melissa W. Chun \\ Dept. of Ophthalmology \\ UCLA \\ Los Angeles, USA \\ chun@jsei.ucla.edu
}

\author{
Clinton Thodos \\ School of Management \\ UCLA \\ Los Angeles, USA \\ clintthodos@ucla.edu
}

\begin{abstract}
More than one million people in the US suffer from hemianopia, which blinds the vision in one half of the peripheral vision in both eyes. Patients with hemianopia are often not aware of what they cannot see and frequently bump into walls, trip over objects, or walk into people on the side where the peripheral vision is diminished. In this study, we present an augmented reality based assistive technology that expands the peripheral vision of hemianopic patients at all distances. In a pilot trial, we evaluate the utility of this assistive technology for ten hemianopic patients. We measure and compare outcomes related to target identification and visual search in the participants. Improvements in target identification are noted in all participants ranging from $18 \%$ to $72 \%$. Similarly, all the participants benefit from the assistive technology in performing a visual search task with an average increase of $24 \%$ in the number of successful searches compared to unaided trials. The proposed technology is the first instance of an electronic vision enhancement tool for patients with hemianopia and is expected to maximize the residual vision and quality of life in this growing, yet largely overlooked population.
\end{abstract}

\section{CCS CONCEPTS}

- Human-centered computing

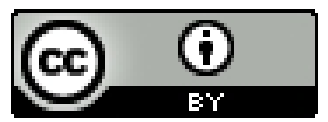

This work is licensed under a Creative Commons Attribution International 4.0 License.

ISWC '20, September 12-16, 2020, Virtual Event, Mexico. (C) 2020 Copyright is held by the owner/author(s).

ACM ISBN 978-1-4503-8077-5/20/09.

https://doi.org/10.1145/3410531.3414296

\section{KEYWORDS}

Hemianopia; Stroke; Assistive Technologies; Augmented Reality

\section{ACM Reference Format:}

Navid Amini, Jung S. Lim, Farnaz Mohammadi, Clinton Thodos, Benjamin Braun, Hassan Ghasemzadeh, Melissa W. Chun, and Kouros Nouri-Mahdavi. 2020. Design and Evaluation of a Wearable Assistive Technology for Hemianopic Stroke Patients. In Proceedings of the 2020 International Symposium on Wearable Computers (ISWC'20), September 12-16, 2020, Virtual Event, Mexico. ACM, New York, NY, USA, 4 pages. https://doi.org/10.1145/3410531.3414296

\section{INTRODUCTION}

Hemianopia affects more than one million people in the United States and is known to interfere with activities of daily living. Hemianopia impairs vision in the left or right half of the visual field in both eyes. The most common cause of hemianopia is stroke [1], followed by traumatic brain injury [2], brain tumors, epilepsy surgery such as hemispherectomy [3] and others. As the population ages and stroke patients live longer, the incidence of stroke and the resultant hemianopic visual field disorders are likely to rise. On the other hand, the emergence and spread of COVID-19 are an added contributing factor that can significantly aggravate the incidence of stroke, as it has been known to cause blood clots that can lead to stroke[4].

The most common type of hemianopia among survivors of stroke and brain injury is homonymous hemianopia, which consists of loss of half the visual field in both eyes on the same side. Unlike many motor and cognitive impairments caused by focal brain injury, spontaneous improvement in hemianopia is rare [1] especially if the hemianopia is complete, i.e., the entire half of the visual field is severely impaired. Hemianopic patients are not able to independently perform their daily activities. People with hemianopia are often not 
aware of what they cannot see and frequently bump into walls, trip over objects or walk into people on the side where the visual field is missing or diminished. Patients with hemianopia are at greater risk of falling [5].

Independent navigation for patients with hemianopia can also be extremely difficult due to their inability to recognize and avoid obstacles. Recent driving simulation tests found that drivers with hemianopia are more likely to hit pedestrians than those with intact visual field [6].

In order to expand the visual field in patients with hemianopia, various optical devices in the form of mirrors, telescopes, and prisms have been proposed [7]. While such devices are commonly prescribed for homonymous hemianopia to expand the visual field, they are known to cause troubling visual side effects, including illusory (nonveridical) location of perceived images, double vision, and visual confusion.

To date, the advantage of programmable, wearable head mounted displays (HMDs) for hemianopia has received minimal attention. Although HMD solutions for the visually impaired were proposed by many researchers, such low-vision aids have had only limited success and are usually not recommended for use by patients in mobile scenarios, such as walking in a hallway or on a sidewalk. The contribution of this work is twofold: i) we utilize the image/video processing power of programmable HMDs equipped with highresolution cameras to provide hemianopic patients with supplemental vision information and widen their available field of vision. Unlike current low-vision aids, which are typically customized for a specific task, the proposed low-vision assistive technology can be used in a variety of settings; ii) we perform a pilot trial where we investigate the functional utility of the proposed technology for hemianopic patient's visual scanning and visual search in mobile and stationary activities.

To the best of our knowledge, the proposed technology is the first instance of an electronic vision enhancement tool for the growing population of hemianopic patients. Based on promising results from ten participants with hemianopia, we expect this technology will significantly improve quality of life of the affected patients and make them less dependent.

\section{RELATED WORK}

We discuss related work from two directions of research: approaches to optical low-vision aids and digital low-vision assistive technologies. Optical Low-Vision Aids. An approach to fitting prisms for homonymous hemianopia was proposed by Eli Peli at Harvard university [8]. The 'Peli lens' is marketed by Chadwick Optical (Souderton, PA) [9]. High-power Fresnel prism segments are placed across the upper and lower peripheral portion of the lens in prescription glasses. Patients with binocular vision experience double vision when viewing through the prisms; nevertheless, they will be able to notice objects in the hemianopic field. Furthermore, these prisms only affect the field-of-view seen simultaneously by both eyes of the patient and they do not affect patients' monocular visual field. Lastly, the prisms cannot easily be adapted and customized to the state of the user (e.g., adaptation to the ambient lighting conditions or customization of the visual field width).

Digital Low-Vision Assistive Technologies. Electronic or digital low-vision aids can be divided into two categories based on their function: those that translate visual information into alternative sensory information, such as sound, vibration, or touch [6]; and those that boost the visibility of visual information and render it in a customized fashion to individuals with low-vision [10], for instance, through contrast enhancements, magnification, or edge enhancement [11]. The emerging technology of wearable HMDs belongs to the second category by utilizing advanced image processing techniques to improve visual experience in a hands-free format.

\section{METHODS}

In this section, we describe the system design as well as the formulation of the trials for our pilot study.

\subsection{System Design}

A popular wearable HMD in the research community, namely the Google Glass Enterprise Edition is used. As a successor of the Original Google Glass, the Enterprise version encompasses a low-energy Intel Atom processor, a $780 \mathrm{mAh}$ battery, an 8-megapixel camera, and a $640 \times 360$ optical display. It can work with all types of existing eyewear and it has a larger, transparent display that presents data without requiring users to look away from their usual viewpoints. Furthermore, the Android-based operating system on this programmable platform allows for custom application development.

\subsection{Expansion of Visual Field}

Based on a user selectable visual field expansion method, the technology displaces the missing parts of the visual image and generates and displays supplemental vision information through an intuitive user interface. Figure 1 demonstrates two scenarios where the supplemental visual information provided by the head-mounted display will help hemianopic patients see objects outside their residual field of view. On the left, people on the walking path of the user can be seen so that collision is avoided. On the right, objects on the walking path of the user (traffic lights and signs) can be seen and magnified by the user. The proposed technology enables hemianopic patients to make real-time decisions based on their perception of objects not normally visible to them without turning their eyes or head. The extent of visual field expansion was measured as the horizontal distance (in degrees) between the unaided (no vision aid) vertical hemifield border (midline) and the same point measured with the vision aid. The technology provides a field-of-view expansion of about $20^{\circ}$ across the horizontal midline.

3.2.1 Image Processing Flow. Figure 2 provides a graphical view of the overall image processing flow of the proposed technology. At first, the captured image is corrected for camera distortion; the image will then be cropped based on the pattern of hemianopic visual field loss. Twodimensional translation and scaling will be applied to utilize the entire resolution of the display. Lastly, the image will be projected onto the viewing region of the HMD. The application was developed in Java 
using Android Studio; all the image processing tasks were realized using OpenCV on the Google Glass processor [12].
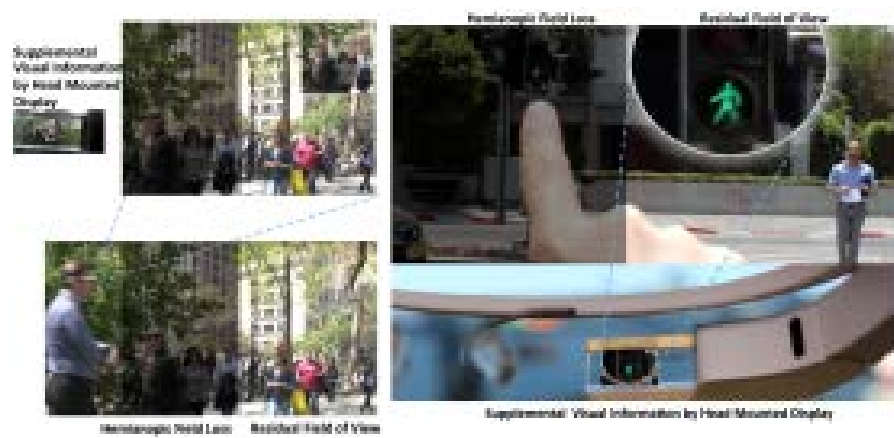

Figure 1. The supplemental visual information provided by the head-mounted display. a) People on the walking path of the user can be seen to avoid a collision. b) Objects on the walking path of the user can be seen and magnified by the user.

3.2.2 Measurement of Head Orientation. We calculate head orientation based on kinematic data from the accelerometer, gyroscope, and magnetometer sensors that are available on the Google Glass. One can estimate the rotation angles that specify the orientation of the head by simply integrating gyroscope readings. However, such measurements suffer from bias (drift), noise, and systematic errors leading to inaccurate orientation estimations [13]. To overcome this issue, we implemented a sensor fusion algorithm to perform gyroscope drift correction by the accelerometer and magnetometer readings. Here we use quaternion-based Extended Kalman Filter (EKF) proposed by Sabatini [14] to estimate the orientation of the head.

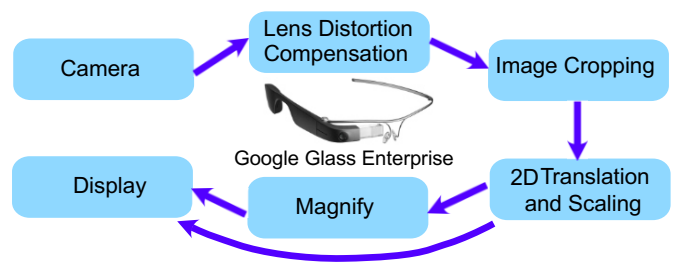

Figure 2. Image processing flow.

\section{Pilot Study}

We conducted an Institutional Review Board (IRB) approved pilot study to evaluate the effectiveness of the low-vision assistive technology through validated tests related to visual scanning (the mobility assessment course) and visual search (the crowded desk test).

\subsection{Participants and Training}

Ten hemianopic patients (five males, five females) are recruited and they perform visual function trials at the UCLA Department of Ophthalmology. Their mean age is $46.7 \pm 18.4$ and the range is from 20 to 74 years. The hemianopic patients are established patients invited by their neuro-ophthalmologist, optometrist, or recruited from several on-campus clinics. Patients are required to have best corrected visual acuity of $20 / 30$ or better in each eye, be able to walk without assistive devices, and not have visual neglect. Furthermore, these ten patients should not have any other concurrent eye disease. During the training, at first, patients are taught to sweep their hand into the hemianopic side and detect it within the display of the HMD. Afterwards, they learn to discriminate targets appearing on the HMD from those on the seeing side and how they should alternate between "augmented vision" and "regular vision". Lastly, the participants will wear the technology for 30 minutes in order to become accustomed to its functionality in a series of small tasks.
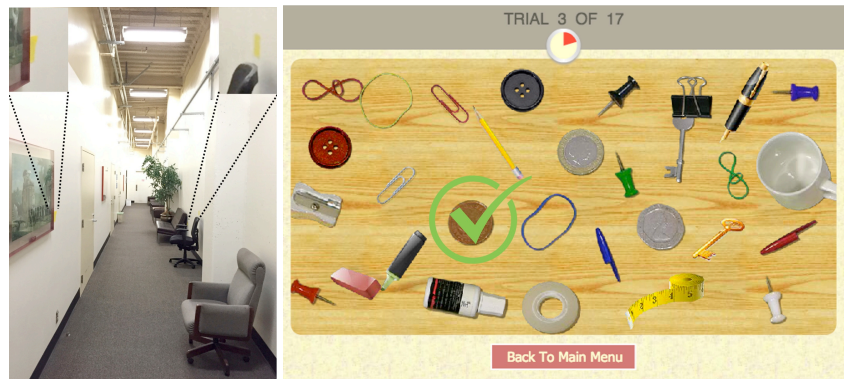

Figure 3. (a) The hallway where the patients perform the mobility assessment course (MAC) with yellow targets placed at different locations on the walls. (b) A screenshot from the crowded desk test for evaluating visual search performance.

\subsection{Evaluation of Visual Scanning Performance}

We conduct the mobility assessment course (MAC) to measure the extent to which patients can visually scan and identify hazards when walking. The MAC has been previously validated in stroke patients [15] and it assesses visual scanning performance on a mobility course with targets placed on the walls (a total of twenty-four yellow targets measuring $100 \mathrm{~mm} \times 100 \mathrm{~mm}$ ) along the course as shown in Figure 3(a). The targets are presented at different heights and against different backgrounds, and are evenly distributed to the left and right sides of space. Participants are instructed to indicate the position of the targets when walking the course. Patents are scored based on the number of targets they identify as measure of obstacle avoidance.

\subsection{Evaluation of Visual Search Performance}

We adapt a validated, reaction time-based, visual search test where patients are required to search for an everyday object in a crowded desk scene. Each trial starts with presentation of a target object in the middle of a screen, which is replaced by the image of a crowded desk (See Figure 3(b)). Search time is defined as the elapsed time from the instant the crowded desk appeared to when the participant located the target object. Each test consists of 17 trials and the number of correct searches with reaction time less than 5 seconds is recorded as the visual search score. Average reaction time for successful searches is also recorded.

\section{RESULTS}

The participants complete three rounds (unaided and aided) of the mobility assessment course (MAC) and the crowded desk tests to evaluate the utility of the low-vision assistive technology. Visual function performance is measured before the participants wear the technology (unaided performance) and while wearing the technology (aided performance) and the results are compared. Participants are allowed to practice before the formal testing and also, they will experience a different version of each test to reduce the learning effect. 
Improvements in target identification, evaluated by MAC test are noted in all participants from unaided trials to aided trials ranging from $18 \%$ to $72 \%$. The number and percentage of targets detected range from $8(33.3 \%)$ to $15(62.5 \%)$ in unaided trials and from $11(45.8 \%)$ to 20 $(83.3 \%)$ in aided trials. Figure 4 indicates significant improvement $(\mathrm{P}<0.001)$ in target identification for all the participants in the mobility assessment course. The results from the crowded desk test reveal average improvements of $24 \%$ in the visual search task in aided trials comparing to unaided ones. Similar to the MAC test, all patients benefit from the assistive technology (see Table I) and the search score significantly improve from unaided trials to aided trials $(\mathrm{P}<0.001$ in paired t-test).

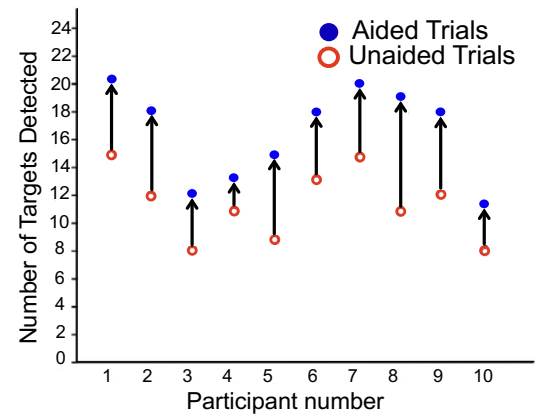

Figure 4. Improvements in target identification from unaided to aided trials in the mobility assessment course with use of HMD.

An important aspect of visual search performance is the speed at which patients complete their search for an object. Therefore, we are interested to see whether patients' trial completion times are significantly altered in aided trials. We find that use of assistive technology improved the average search time in three patients; however, the majority of the participants experience a slightly slower search time (by $0.4 \mathrm{~ms}$ on average).

Table I. Summary of Search Performance in Crowded Desk Test

\begin{tabular}{|c|c|c|}
\hline Participant \# & $\begin{array}{c}\text { Search Score (Unaided, } \\
\text { Aided) }\end{array}$ & $\begin{array}{c}\text { Average Search } \\
\text { Time (Unaided, } \\
\text { Aided) }\end{array}$ \\
\hline 1 & $7(41 \%), 12(71 \%)$ & $3.4 \mathrm{~s}, 4.4 \mathrm{~s}$ \\
\hline 2 & $8(47 \%), 14(82 \%)$ & $3.5 \mathrm{~s}, 3.4 \mathrm{~s}$ \\
\hline 3 & $10(59 \%), 12(71 \%)$ & $4.2 \mathrm{~s}, 4.5 \mathrm{~s}$ \\
\hline 4 & $9(53 \%), 16(94 \%)$ & $3.4 \mathrm{~s}, 3.4 \mathrm{~s}$ \\
\hline 5 & $6(35 \%), 9(53 \%)$ & $4.4 \mathrm{~s}, 4.9 \mathrm{~s}$ \\
\hline 6 & $10(59 \%), 12(71 \%)$ & $3.8 \mathrm{~s}, 4.1 \mathrm{~s}$ \\
\hline 7 & $9(53 \%), 10(59 \%)$ & $2.9 \mathrm{~s}, 2.8 \mathrm{~s}$ \\
\hline 8 & $11(65 \%), 16(94 \%)$ & $3.5 \mathrm{~s}, 3.2 \mathrm{~s}$ \\
\hline 9 & $7(41 \%), 10(59 \%)$ & $4.1 \mathrm{~s}, 4.3 \mathrm{~s}$ \\
\hline 10 & $6(35 \%), 13(76 \%)$ & $4.1 \mathrm{~s}, 4.5 \mathrm{~s}$ \\
\hline
\end{tabular}

\section{DISCUSSION}

We propose an assistive technology utilizing data intensive image processing techniques that produce unambiguous and timely spatial information in the form of successive frames displayed in real time on the screen of the HMD to widen hemianopic patients' available field of vision. Furthermore, in a small IRB-approved pilot trial, we investigate whether our proposed assistive technology and its supplemental visual information can improve patients' visual function.

Through the MAC test, we find that all recruited patients in the study benefit from the assistive technology when performing target identification tasks in a mobile scenario. Another finding of the study is that the assistive technology improves the visual search performance in the entire patient cohort, albeit with a slightly increased search time in the majority of the participants. This increased search time could be attributed to the time spent on switching between regular vision and the augmented vision provided by the assistive technology. We report the results of the pilot trial based on Google Glass Enterprise Edition; however, our Androidbased mobile application is platform independent and with slight modifications it can run on multiple head-mounted display platforms such as Epson Moverio BT-300, Vuzix M-300 and newer versions of Google Glass (e.g., Google Glass Enterprise Edition 2).

This study proposes a platform for developing a vision enhancement tool for patients with visual field defects that may have resulted from other vision disorders, such as anterior ischemic optic neuropathy (AION), retinitis pigmentosa, and glaucoma. Unlike current low-vision aids, which are typically customized for a specific task, the proposed low-vision assistive technology can be used in a variety of settings (in-home, outdoor, mobile, stationary, etc.).

\section{CONCLUSIONS}

In this study, we propose an augmented reality based assistive technology to provide hemianopic patients with supplemental visual information and to widen their available field of vision. We demonstrate that our assistive technology improves visual function in both mobile and stationary tasks such as target identification and visual search in a pilot trial involving ten hemianopic patients. Our proposed assistive technology will identify solutions for mobility training as well as independent living rehabilitation in the growing hemianopia population.

\section{AKNOWLEDGEMENTS}

We would like to greatly thank Vodafone Americas Foundation for providing us with financial support for this study.

\section{REFERENCES}

[1] X. Zhang, S. Kedar, M. J. Lynn, N. J. Newman, and V. Biousse, "Homonymous hemianopia in stroke," Journal of Neuro-Ophthalmology. 2006.

[2] B. B. Bruce, X. Zhang, S. Kedar, N. J. Newman, and V. Biousse, "Traumatic homonymous hemianopia," J. Neurol. Neurosurg. Psychiatry, 2006.

[3] A. N. V Moosa et al., "Long-term functional outcomes and their predictors after hemispherectomy in 115 children," Epilepsia, vol. 54, no. 10, pp. 1771-1779, 2013.

[4] and M. B. Markus, Hugh S., "COVID-19 and stroke-A global World Stroke Organization perspective,” Int. J. stroke, vol. 15, no. 4, pp. 361-364, 2020.

[5] D. Goodwin, "Homonymous hemianopia: challenges and solutions," Clin. Ophthalmol., vol. 8, pp. 1919-1927, 2014.

[6] A. R. Bowers, E. Ananyev, A. J. Mandel, R. B. Goldstein, and E. Peli, "Driving with hemianopia: IV. head scanning and detection at intersections in a simulator," Investig. Ophthalmol. Vis. Sci., 2014. 
[7] A. R. Lane, D. T. Smith, and T. Schenk, "Clinical treatment options for patients with homonymous visual field defects.," Clin. Ophthalmol., vol. 2, no. 1, pp. 93-102, 2008.

[8] E. Peli, "Field expansion for homonymous hemianopia by optically induced peripheral exotropia.," Optom. Vis. Sci., vol. 77, no. 9, pp. 453-64, 2000

[9] Chadwick Optical Inc., "Showing a 'Peli lens' fitting kit and press-on prisms." [Online]. Available: http://chadwickoptical.com/peli-lens-2/.

[10] H. S. van Rheede JJ, Wilson IR, Qian RI, Downes SM, Kennard C, "Improving Mobility Performance in Low Vision With a Distance-Based Representation of the Visual Scene," Invest Ophthalmol Vis Sci., vol. 56, no. 8, pp. 4802-9, 2015.

[11] A. D. Hwang and E. Peli, "An augmented-reality edge enhancement application for Google glass,” Optom. Vis. Sci., 2014.

[12] D. Jelena, "Developing OpenCV applications on Google Glass 2," University of the Balearic Islands, 2018.

[13] M. Li, H. Yu, X. Zheng, and A. I. Mourikis, "High-fidelity sensor modeling and selfcalibration in vision-aided inertial navigation," in Proceedings - IEEE International Conference on Robotics and Automation, 2014.

[14] A. M. Sabatini, "Quaternion-based extended Kalman filter for determining orientation by inertial and magnetic sensing," IEEE Trans. Biomed. Eng., 2006.

[15] D. Verlander et al., "Assessment of clients with visual spatial disorders: A pilot study,” Vis. Impair. Res., 2000. 